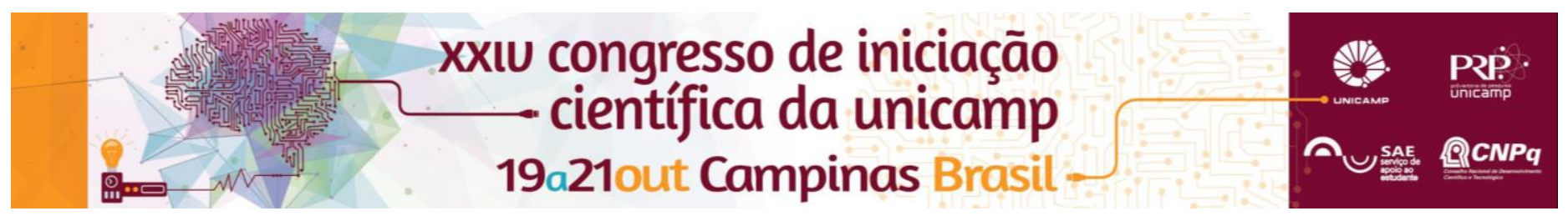

\title{
Analysis of continuous flight auger behaviour in sedimentary soil
}

\author{
Jéssica V. Curci*, Paulo J. R. Albuquerque.
}

\begin{abstract}
This project studies the behaviour of continuous flight auger in a sedimentary soil, submitted to static compression loading tests. There are two different locals being analysed, CFA lengths varying between 10 and $13 \mathrm{~m}$. The whole process is instrumented, and the instrumentation consists of electrical strain gages sticked to steel bars, installed in different levels along the CFA
\end{abstract}

\section{Key words:}

Continuous Flight Auger (CFA), compression loading tests, instrumentation

\section{Introduction}

CFA are becoming more and more important in construction field in Brazil, for medium and large works. Because of that, researches that can help to understand the interactions soil-pile are considered relevant. This research analyses two different static compressionloading tests, in order to obtain some parameters to analyse the soil-pile relation. The objective is to obtain the load $x$ settlement and load transferring curves, in order to compare the obtained results with theoretical and semiempirical methods.

\section{Results and Discussion}

During this research, seven methods were studied. Two different theoretical methods: Terzaghi (1943) and Meyerhof (1976), and five semi-empirical methods: Aoki \& Velloso (1978), Décourt \& Quaresma (1978/1996), Teixeira (1996), Alonso (1996), Vorcaro \& Velloso (2000). The theoretical results were obtained by correlations of soil's parameters, as the semi-empirical results were obtained by the data given by SPT. The results obtained during the static compression-loading tests were extrapolated using three different methods: Chin (1970), Decourt (1996) and Van der Veen (1953) modified by Aoki (1976). In results, a great difference between methods can be noticed. Some of the results showed less pile capacity then real, while others showed higher capacity.

\section{Chart 1. Results and relation between methods - Local} B

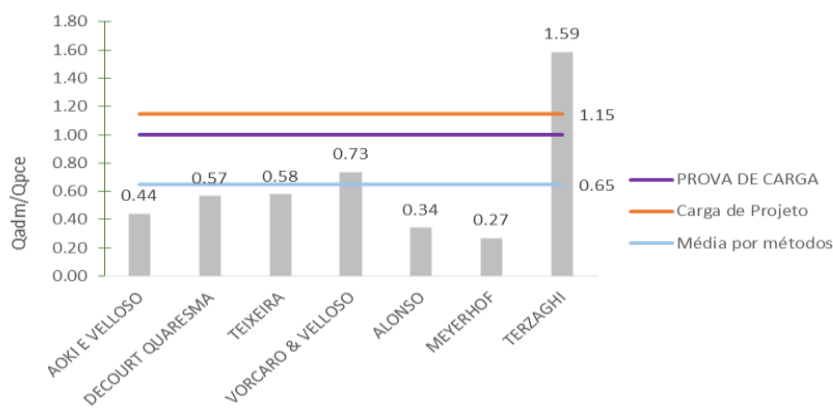

The research also tries to understand load how the load is transferred during the pile length and how lateral capacity can absorb loads before they reach the bottom of the pile.

\section{Image 2. Load transference - Locals A}

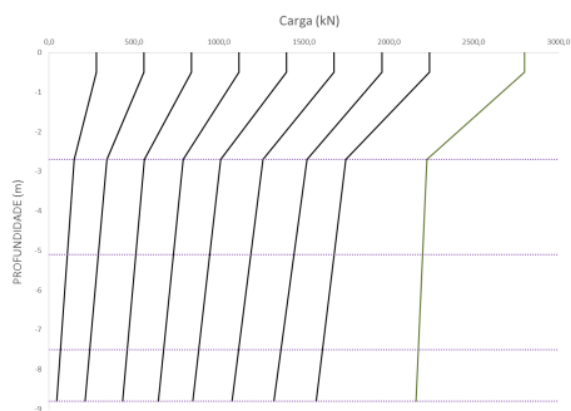

\section{Conclusions}

All methods can fail, underestimating or overestimating results. There is no rule: In some locals, a method can overestimate, while at other local, it can underestimate. The theoretical methods usually are less reliable, because they carry many mistakes of correlations, and also because they don't consider the difference between piles or between their execution.

At this research, the results obtained by theoretical and semi empirical methods represent only $70 \%$ of the real result obtained by the load test. It means the methods underestimated the pile capacity, and probably more money was spent unnecessarily, to guarantee safety.

It is possible to say that the best method to obtain a pile's capacity is the compression-loading test.

During this research, it was also possible to verify that depending on the soil, more or less load will be absorbed by the lateral area.

CINTRA, J. C. A; AOKI, N.; Carga Admissível em Fundação Profunda .1.ed. São Carlos, 1999, Editora RIMA,

DAS, B. M., Principles of Foundation Engineering, 6th ed., United States of America: Thomson Canada Limited, 2007. 